Pacific Journal of Mathematics

SOME INEQUALITIES BETWEEN LATENT ROOTS AND 


\section{SOME INEQUALITIES BETWEEN LATENT ROOTS AND MINIMAX (MAXIMIN) ELEMENTS OF REAL MATRICES}

LOUIS GUTTMAN

1. Introduction. Because of the usual tediousness of computing latent roots, any quick information about them is often welcome and useful. We develop here some lower bounds to the absolute value of the major latent root (the one largest in absolute value) of any real symmetric matrix that depend only on a simple inspection of its elements. Also, lower bounds are developed for the largest latent root of a Gramian matrix of the form $A A^{\prime}$ that require inspection only of the elements of $A$. The latter case is especially important in linear regression theory of statistics, in factor analysis theories of psychology, and elsewhere.

The original motivation for our inequalities was to study the relationship between latent roots and the von Neumann value of a two-person zero-sum game matrix. We actually use the von Neumann theory to establish our bounds to latent roots, and in return we show how latent roots can be used to bound the game value of a matrix. The latter kind of bound will be useful whenever it is easier to get at the appropriate latent root than at the desired game value.

The bounds to latent roots are first exhibited in $\S \S 2-3$, and then proved in $\S 4$. How to reverse their emphasis to provide bounds for game values is shown in $\S 5$.

2. Lower bounds to the major latent root. Let $A$ be any real matrix of order $m \times n$. Let $a_{i j}$ be the typical element of $A(i=1,2$, $\cdots, m ; j=1,2, \cdots, n)$, and let $p_{i}$ and $q_{j}$ be defined respectively as

$$
p_{i}=\min _{j} a_{i j}, \quad q_{j}=\max _{i} a_{i j} \quad\left(\begin{array}{l}
i=1,2, \cdots, m \\
j=1,2, \cdots, n
\end{array}\right) .
$$

Furthermore, let $p$ and $q$ be defined respectively as

$$
p=\max _{i} p_{i}, \quad q=\min _{j} q_{j} .
$$

From (1), it immediately follows that

$$
p_{i} \leqq a_{i j} \leqq q_{j} \quad\left(\begin{array}{c}
i=1,2, \cdots, m \\
j=1,2, \cdots, n
\end{array}\right),
$$

Received February 20, 1956. 
and in particular that $p \leqq q$.

Let $\lambda^{2}$ be the largest latent root of $A A^{\prime}$, where $A^{\prime}$ is the transpose of $A$. We shall prove in $\S 4$ below that both of the following inequalities hold :

$$
\begin{aligned}
& |\lambda| \geqq p \sqrt{n} \\
& |\lambda| \geqq-q \sqrt{m} .
\end{aligned}
$$

Inequality (4) is a useful lower bound to $|\lambda|$ if and only if $p>0$, while (5) is useful if and only if $q<0$. If $p \leqq 0 \leqq q$, we obtain no information about $|\lambda|$.

One interesting feature of (4) and (5) is that they show that $\lambda^{2}$ is generally at least of the order of $m$ or of $n$, depending on whether $q<0$ or $p>0$.

Corresponding inequalities can be developed by considering $A^{\prime}$ in place of $A$. Let $p_{j}^{\prime}$ and $q_{i}^{\prime}$ be defined respectively as

$$
p_{j}^{\prime}=\min _{i} a_{i j}, \quad q_{i}^{\prime}=\max _{j} a_{i j} \quad\left(\begin{array}{c}
i=1,2, \cdots, m \\
j=1,2, \cdots, n
\end{array}\right),
$$

so that

$$
p_{j}^{\prime} \leqq a_{i j} \leqq q_{i}^{\prime}
$$$$
\left(\begin{array}{l}
i=1,2, \cdots, m \\
j=1,2, \cdots, n
\end{array}\right)
$$

Let $p^{\prime}$ and $q^{\prime}$ be defined by

$$
p^{\prime}=\max _{j} p_{j}^{\prime} . \quad q^{\prime}=\min _{i} q_{i}^{\prime},
$$

whence, from (7), $p^{\prime} \leqq q^{\prime}$.

Now, $A A^{\prime}$ and $A^{\prime} A$ have the same nonzero latent roots, which are all positive. So if $\lambda^{2}$ is the largest latent root of $A A^{\prime}$, it is also the largest latent root of $A^{\prime} A$. In addition to (4) and (5), we can write

$$
\begin{aligned}
& |\lambda| \geqq p^{\prime} \sqrt{m} \\
& |\lambda| \geqq-q^{\prime} \sqrt{n} .
\end{aligned}
$$

Notice that the roles of $m$ and $n$ in (9) and (10) are reversed from those in (4) and (5). If $p^{\prime}>0, \lambda^{2}$ is at least of order $m$, while if $q^{\prime}<0$, $\lambda^{2}$ is at least of order $n$. If either of $p$ or $p^{\prime}$ is positive, or if either of $q$ or $q^{\prime}$ is negative, we get some information about $|\lambda|$.

Matrices of the form $A A^{\prime}$ or $A^{\prime} A$ are called Gramian, or nonnegative definite symmetric. In statistics, any correlation matrix $R$ is Gramian. A good deal of work in psychology, for example, is aimed at "factoring" an $R$ into the form $R=A A^{\prime}$. Given such a factoring, our inequalities 
immediately given lower bounds to the largest latent root of $R$ from the minimax and maximin element of $A$. The latter are easily ascertainable by inspection.

3. The case of symmetric matrices. If $m \neq n, A$ itself has no latent roots defined. However, if $A$ is square, then it does have a characteristic equation and latent roots. A particularly important case is where $A$ is symmetric, or $A=A^{\prime}$. Then the latent roots of $A$ are all real, and their squares are the latent roots of $A A^{\prime}=A^{2}$. If $\lambda^{2}$ is the largest latent root of $A A^{\prime}$, then $\lambda$ must be a root of $A$ largest in absolute value, and conversely. In this symmetric case, we have not only $m=n$, but also $p=p^{\prime}, q=q^{\prime}$. So (9) and (4) are redundant, as are also (10) and (5). The inequalities can now be interpreted as referring to the major latent root of $A$ itself, and not merely to a root of $A A^{\prime}$.

When $A$ is symmetric, we can usually improve on (4) and (5).

Let $I$ be the unit matrix of order $n, c$ be an arbitrary constant, and $A^{*}$ be defined as

$$
A^{*}=A-c I
$$

If $\lambda$ is a latent root of $A$, then $\lambda-c$ is a latent root of $A^{*}$, and conversely. Let $p^{*}$ and $q^{*}$ be the maximin and minimax of elements of $A^{*}$ respectively, or, if $\delta_{i j}$ is Kronecker's delta,

$$
p^{*}=\max _{i} \min _{j}\left(a_{i j}-c \delta_{i j}\right), \quad q^{*}=\min _{i} \max _{j}\left(a_{i j}-c \delta_{i j}\right) .
$$

Then in place of (4) and (5), we can write

$$
|\lambda-c| \geqq p^{*} \sqrt{n}, \quad|\lambda-c| \geqq-q^{*} \sqrt{n} \quad\left(A=A^{\prime}\right),
$$

where $\lambda-c$ is the major latent root of $A^{*}$. In special cases, a judicious choice of $c$ may be apparent that will make maximum $|\lambda-c|$ correspond to a $\lambda$ which is either the most positive or the most negative latent root of $A$, and with a better bound than given by (4)-(5).

An especially important symmetric case is where $A$ is a correlation matrix $R$, with all diagonal elements equal to unity. In such a case, the largest latent root of $R$ cannot be less than 1 , for the trace of $R$ is $n$ and all $n$ latent roots are nonnegative. For this case, if $p>0$, then choose $c=1-p$. This implies that the main diagonal elements of $R^{*}$ are all equal to $p$. Then, clearly $p=p^{*}$; and since $\lambda \geqq 1$ for any $R$, $|\lambda-1+p|=\lambda-1+p$ when $p>0$, and (13) becomes

$$
\lambda \geq 1+p(\sqrt{ } n-1) \quad(p \geqq 0, A=R) .
$$

Similarly, if $q<0$, by choosing $c=1-q$ in (13) we get 


$$
\lambda \geq 1-q(\sqrt{n}-1) \quad(q \leqq 0, A=R) .
$$

4. Proof of the inequalities. Let $P_{k}$ denote the space of all $k$ dimensional probability row vectors. That is $z \in P_{k}$ if and only if $z$ is a row of $k$ nonnegative numbers whose sum equals unity. Let $z^{\prime}$ denote the column vector that is the transpose of $z$. Then $z z^{\prime}$ is the sum of squares of the components of $z$, and it is easily established and wellknown that

$$
\frac{1}{k} \leqq z z^{\prime} \leqq 1 \quad\left(z \in P_{k}\right)
$$

The equality on the left of (16) is always attained by letting $z=z_{1}$, where $z_{1}$ is a vector whose components all equal $1 / k$ (and hence $z_{1} \in P_{k}$ ).

von Neumann [1] has shown how each real matrix $A$ has associated with it a unique real number $v$ with certain important minimax properties. Since his theorem was developed in the context of his theory of games, we shall call $v$ the game value of $A$. Our present interest of course is to regard von Neumann's theorem as a general theorem on real matrices, without necessary reference to the theory of games.

von Neumann's theorem is as follows. If $A$ is a real matrix of order $m \times n$, then there exist an $x_{0}$ and $a y_{0}$, where $x_{0} \in P_{m}$ and $y_{0} \in P_{n}$, and a unique real number $v$, such that

$$
x A y_{0}^{\prime} \leqq v \leqq x_{0} A y^{\prime} \quad \text { for all } \quad x \in P_{m}, \quad y \in P_{n} .
$$

Furthermore,

$$
p \leqq v \leqq q,
$$

where $p$ and $q$ are as defined in (2).

To use this theorem for establishing our own inequalities, apply Schwarz's inequality to (17) to see that

$$
-\sqrt{\left(x x^{\prime}\right)\left(y_{0} A^{\prime} A y_{0}^{\prime}\right) \leqq v \leqq} \sqrt{\left(y y^{\prime}\right)\left(x_{0} A A^{\prime} x_{0}^{\prime}\right)} \quad\left(x \in P_{m}, y \in P_{n}\right) .
$$

Let $\lambda^{2}$ be the largest latent root of $A A^{\prime}$ and $A^{\prime} A$. Then

$$
x_{0} A A^{\prime} x_{0}^{\prime} \leqq \lambda^{2} x_{0} x_{0}^{\prime} \leqq \lambda^{2}, \quad y_{0} A^{\prime} A y_{0}^{\prime} \leqq \lambda^{2} y_{0} y_{0}^{\prime} \leqq \lambda^{2},
$$

the second inequalities in each part of (20) following from the second inequality in (16). From the first inequality in (16),

$$
x x^{\prime} \geqq \frac{1}{m}, \quad y y^{\prime} \geqq \frac{1}{n} \quad\left(x \in P_{m}, y \in P_{n}\right),
$$

and we have noted that the equalities in (21) are always attainable, by best possible $x_{1}$ and $y_{1}$ for this purpose. Using (20) and the equalities 
of (21) in (19) yield

$$
-|\lambda| \leqq v \leqq \frac{|\lambda|}{\sqrt{ } m}
$$

Then (4) and (5) follow from (22) and (18). Inequalities (9) and (10) follow from the restatement of (22) for the game value $v^{\prime}$ of $A^{\prime}$ :

$$
\frac{-|\lambda|}{\sqrt{n}} \leqq v^{\prime} \leqq \frac{|\lambda|}{\sqrt{m}}
$$

Inequalities (22) and (23) are of course sharper than those stated in $\S 2$ above. If game values are known, they can be used in place of $p$, $q, p^{\prime}$, or $q^{\prime}$ in the latter inequalities. We have stated our inequalities in the form most practical to use, since $p$ and $q$ can be determined by inspection, whereas $v$ usually cannot, except in the special case where $p=q=v$.

5. Application to game values. Let us now consider the converse problem of bounding game values. If an upper bound to $|\lambda|$ is known, this will serve to bound $v$ and $v^{\prime}$ via (22) and (23). Thus, useful bounds to $v$ can be set that may sometimes be better than (18) when $p \neq q$. Perhaps more important, (22) and (23) show how the magnitudes of $v$ and $v^{\prime}$ compare with those of $m$ and $n$ in general, given some notion of the size of $|\lambda|$.

For the purpose of bounding $v$ and $v^{\prime},(22)$ and (23) can be improved on. Let $A_{c}$ be the $m \times n$ matrix whose typical element is $a_{i j}-c$, where $c$ is an arbitrary constant. Thus $A_{c}$ is obtained by subtracting $c$ from each element of $A$ (so $A_{c} \neq A^{*}$ if $c \neq 0$ ). It is easily verified that the game value of $A_{c}$ is $v-c$, and optimal probability vectors $x_{0}$ and $y_{0}$ for $A$ are optimal also for $A_{c}$. Let $\lambda_{c}^{2}$ be the largest latent root of $A_{c} A_{c}^{\prime}$ (or of $A_{c}^{\prime} A_{c}$ ). Then we can replace (22) and (23) by the more general inequalities

$$
c-\frac{\left|\lambda_{c}\right|}{\sqrt{m}} \leqq v \leqq c+\frac{\left|\lambda_{c}\right|}{\sqrt{n}}
$$

and

$$
c-\frac{\left|\lambda_{c}\right|}{v n} \leqq v^{\prime} \leqq c+\frac{\left|\lambda_{c}\right|}{\sqrt{m}}
$$

Evidently, the best choice of $c$ is that which will minimize $\lambda_{c}^{2} . A$ practical way to approximate this choice is to minimize instead the sum of all the latent roots of $A_{c} A_{c}^{\prime}$, or the trace of $A_{c} A_{c}^{\prime}$. This requires 
minimizing

$$
\sum_{i=1}^{m} \sum_{j=1}^{n}\left(a_{i j}-c\right)^{2},
$$

for which the minimizing value is $c=a$, where

$$
\bar{a}=\frac{1}{m n} \sum_{i=1}^{m} \sum_{j=1}^{n} a_{i j} .
$$

\section{REFERENCE}

1. J. von Neumann and O. Morgenstern, Theory of games and economic behavior, Prin ceton Univ. Press, 1954.

Center for Advanced Study in the Behavioral Sciences (on leave from the Israel Institute of Applied Social ReSEarch) 


\section{PACIFIC JOURNAL OF MATHEMATICS}

EDITORS

H. L. ROYDEN

Stanford University

Stanford, California

R. A. Beaumont

University of Washington

Seattle 5 , Washington
A. R. Whiteman

University of Southern California

Los Angeles 7, California

E. G. Straus

University of California

Los Angeles 24, California

\section{ASSOCIATE EDITORS}
E. F. BECKENBACH
C. E. BURGESS
M. HALL
E. HEWITT
A. HORN
V. GANAPATHY IYER
R. D. JAMES
M. S. KNEBELMAN

L. NACHBIN

I. NIVEN

G. SZEKERES

T. G. OSTROM

M. M. SCHIFFER
F. WOLF

K. YOSIDA

\section{SUPPORTING INSTITUTIONS}

UNIVERSITY OF BRITISH COLUMBIA

CALIFORNIA INSTITUTE OF TECHNOLOGY

UNIVERSITY OF CALIFORNIA

MONTANA STATE UNIVERSITY

UNIVERSITY OF NEVADA

OREGON STATE COLLEGE

UNIVERSITY OF OREGON

UNIVERSITY OF SOUTHERN CALIFORNIA

\author{
STANFORD UNIVERSITY \\ UNIVERSITY OF UTAH \\ WASHINGTON STATE COLLEGE \\ UNIVERSITY OF WASHINGTON \\ AMERICAN MATHEMATICAL SOCIETY \\ CALIFORNIA RESEARCH CORPORATION \\ HUGHES AIRCRAFT COMPANY \\ THE RAMO-WOOLDRIDGE CORPORATION
}




\section{Pacific Journal of Mathematics}

\section{Vol. 7, No. $1 \quad$ January, 1957}

Richard Davis Anderson, Zero-dimensional compact groups of

homeomorphisms ................................... 797

Hans-Joachim Bremermann, Holomorphic functionals and complex

convexity in Banach spaces........................... 811

Hugh D. Brunk, G. M. Ewing and W. R. Utz, Minimizing integrals in

certain classes of monotone functions ................. 833

Philip David, Uniqueness theory for asymptotic expansions in general

regions ...................................... 849

Paul Erdős and Harold Nathaniel Shapiro, On the least primitive root of a

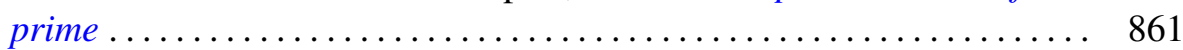

Watson Bryan Fulks, Regular regions for the heat equation ........... 867

William Robert Gaffey, A real inversion formula for a class of bilateral

Laplace transforms ................................ 879

Ronald Kay Getoor, On characteristic functions of Banach space valued random variables ................................. 885

Louis Guttman, Some inequalities between latent roots and minimax (maximin) elements of real matrices ...................... 897

Frank Harary, The number of dissimilar supergraphs of a linear graph .... 903

Edwin Hewitt and Herbert S. Zuckerman, Structure theory for a class of convolution algebras .................................. 913

Amnon Jakimovski, Some Tauberian theorems . . . . . . . . . . . . . . . . . 943

C. T. Rajagopal, Simplified proofs of "Some Tauberian theorems" of Jakimovski................................

Paul Joseph Kelly, A congruence theorem for trees ................. 961

Robert Forbes McNaughton, Jr., On the measure of normal formulas...... 969

Richard Scott Pierce, Distributivity in Boolean algebras .............. 983

Calvin R. Putnam, Continuous spectra and unitary equivalence ......... 993

Marvin Rosenblum, Perturbation of the continuous spectrum and unitary

equivalence................................... 997

V. N. Singh, Certain generalized hypergeometric identities of the

Rogers-Ramanujan type.......................

Peter Swerling, Families of transformations in the function spaces $H^{p} \ldots \ldots 1015$ 